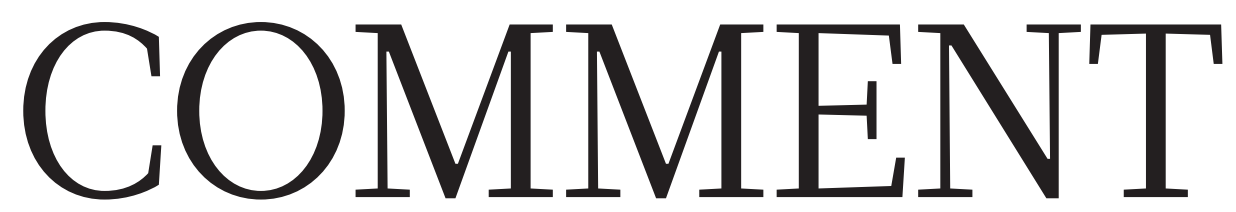

\begin{tabular}{l|l|l|l|l}
\hline LAW CRISPR-Cas9 patent & $\begin{array}{l}\text { Books Tim Birkhead's } \\
\text { suit highlights a troubling } \\
\text { institutional trend p.172 }\end{array}$ & $\begin{array}{l}\text { INVASIVES Stop the yellow- } \\
\text { legged hornet's destructive } \\
\text { march through Europe } \mathbf{p . 1 7 7}\end{array}$ & $\begin{array}{l}\text { OBITUARY Lloyd Shapley, } \\
\text { game-theory Nobel laureate, } \\
\text { remembered p.178 }\end{array}$ \\
\hline
\end{tabular}

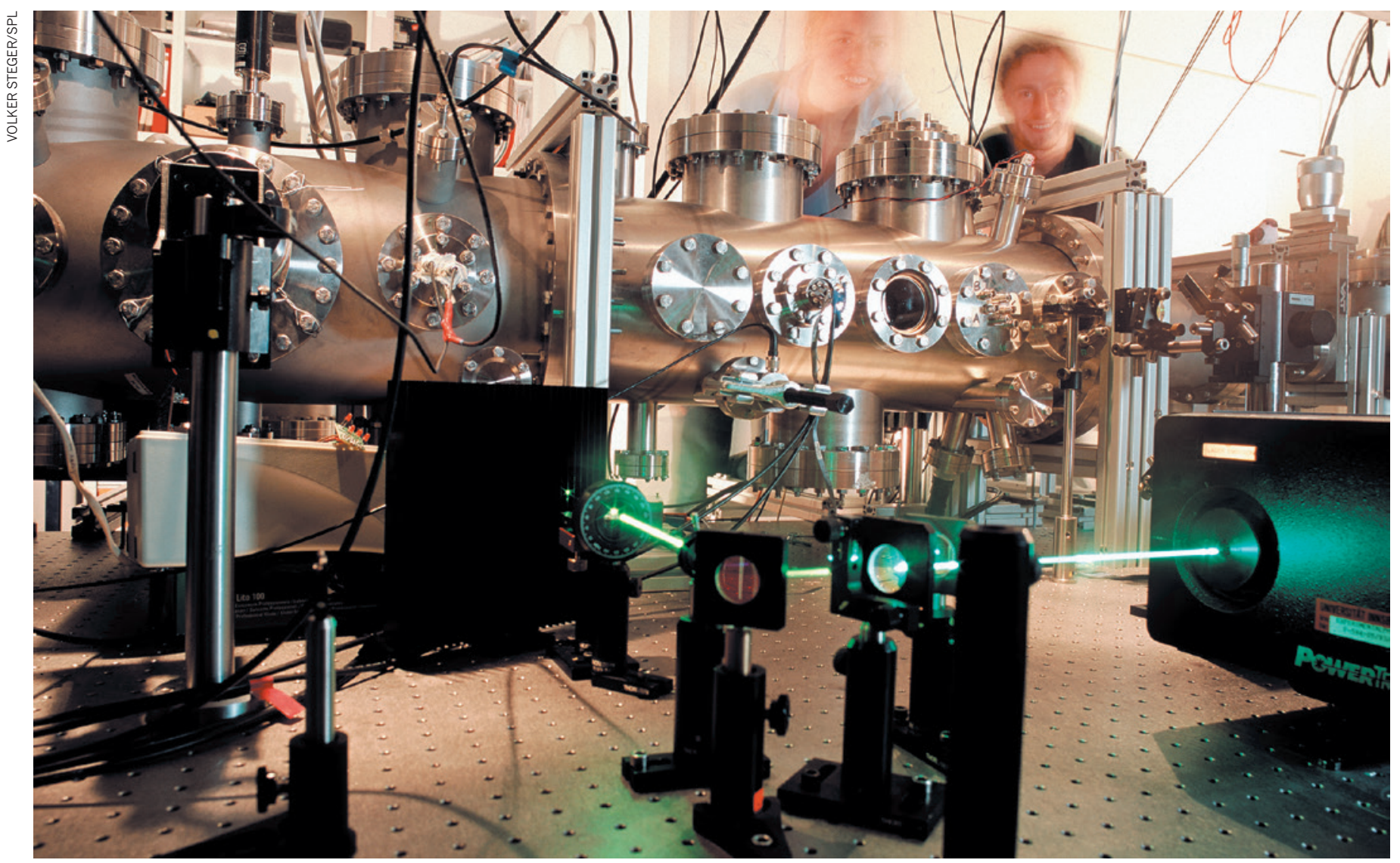

A quantum-entanglement experiment at the University of Vienna in Austria.

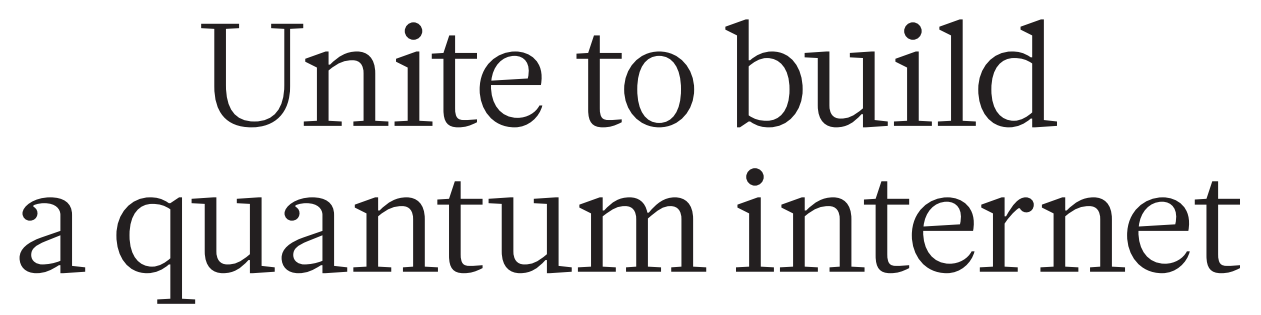

Advances in quantum communication will come from investment in hybrid technologies, explain Stefano Pirandola and Samuel L. Braunstein.

\begin{abstract}
lmost 25 years ago, physicists discovered a way of 'teleporting' a quantum system from one place to another without moving it ${ }^{1}$. There are physical limits to such teleportation: nothing can be transmitted faster than the speed of light; and Heisenberg's uncertainty principle restricts what we can know about the state of a quantum system at a
\end{abstract}

given time. Nevertheless, the transported system is a replica that perfectly mimics the original, thanks to the weirdest feature of quantum mechanics - entanglement. Described by Albert Einstein as "spooky action at a distance", this property enables distinct quantum systems to become intimately correlated so that an action performed on one has an effect on the other, even for systems that are too far apart to physically interact.

Quantum states are fragile and cannot be sent through conventional lines of communication; quantum teleportation offers a reliable and efficient way to transfer quantum information across a network. It provides the most promising mechanism for a future quantum internet, with 
secure communications and a distributed computational power that greatly exceeds that of the classical Internet.

Quantum information comes in a variety of forms - the polarization state of a photon, the spin of an electron or the excitation state of an atom. Many technologies have been developed for teleporting such states ${ }^{2}$. But there are practical restrictions on what can be teleported, and how. Certain technologies will be better than others for particular tasks, and each has its limitations. Polarized photons have been used to transfer quantum information over more than 100 kilometres $^{3}$, but only probabilistically. Superconducting devices can send information without losses through a chip, but only for a split second, after which the information is scrambled by interactions with the environment.

Hybrid approaches might overcome these limitations. A global, distributed quantum computer or internet will need to integrate different sorts of quantum technologies. For example, light-based teleportation, for long-distance quantum communication, will need to be linked to matter-based quantum memories and quantum computers for data storage and data processing. Here, we outline the main challenges and call for researchers to focus on the interfaces between quantum technologies as well as advancing individual methods.

\section{TWO APPROACHES}

The best technique at present for longdistance communication is the teleportation of quantum information that is embedded in optical light. Quantum information measured in units known as quantum bits, or qubits - can be encoded either by the discrete properties of a pulse of light, such as its polarization state, or by the continuous aspects of an electromagnetic wave, such as the intensity and phase of the wave's electric field $^{4}$. To teleport this information, both the sender and receiver must own one of a pair of entangled quantum systems (see 'Quantum teleportation'). When the sender alters the state of their system, the receiver's system is also affected.

Polarization qubits perform best in terms of distance - holding a record of 143 kilometres ${ }^{3}$. But currently, only $50 \%$ of these qubits can be teleported ${ }^{2}$. Teleportation requires that the sender can carry out an operation known as a Bell detection, in which the polarizations of two qubits are correlated perfectly in four possible configurations. But there is no practical way to measure all four outcomes. Simple optics and photodetectors can distinguish two, at most. Extra qubits add technical complications ${ }^{5}$.

Inconclusive outcomes such as these are acceptable for quantum cryptography, in which secret keys are generated at random, and part of the information can be discarded. But quantum communication demands that information is sent in full.

Teleportation over long distances ${ }^{2}$ brings further technical challenges, such as compensating for atmospheric turbulence and movement of the ground. It is also likely to require new technologies to synchronize

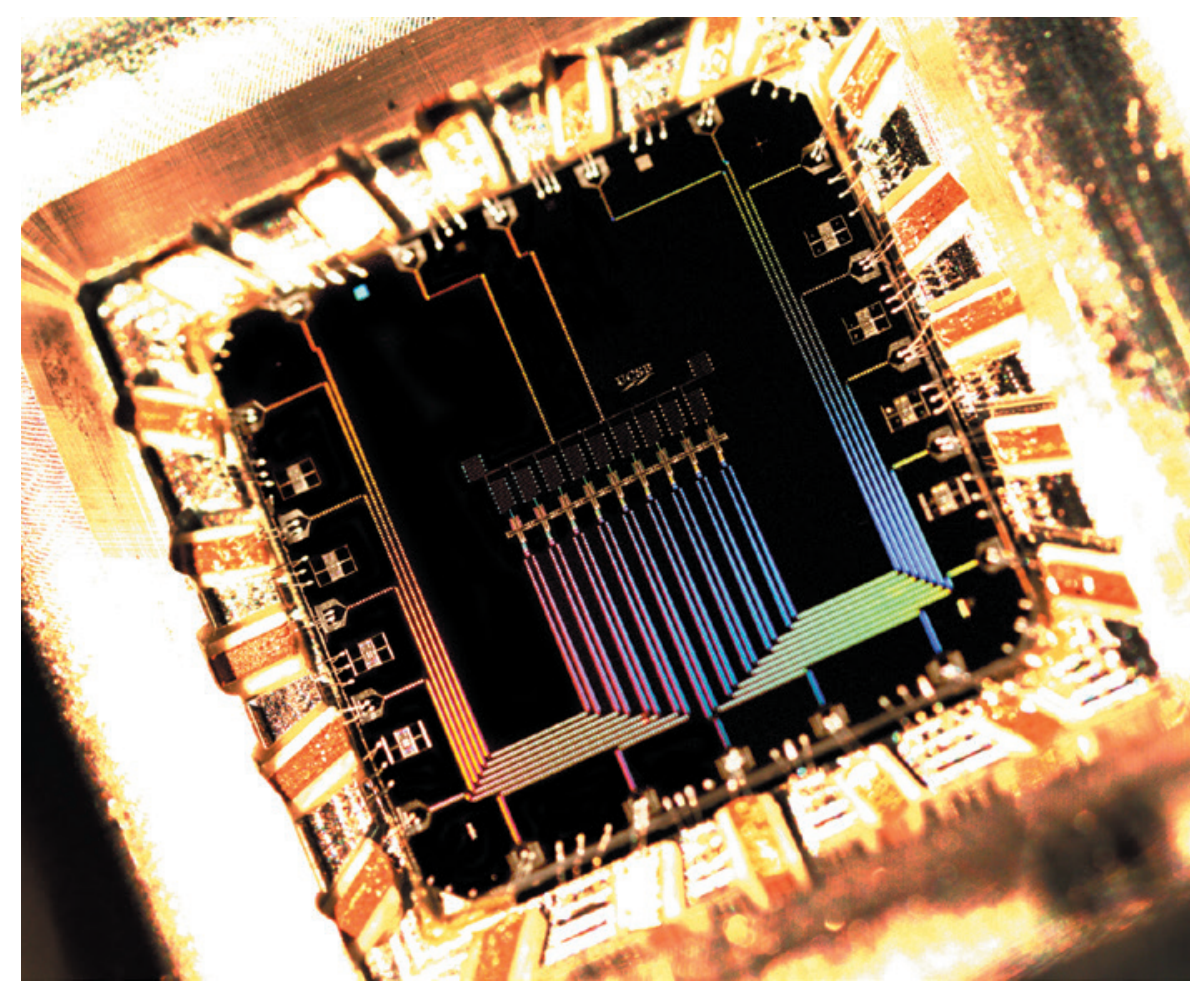

A superconducting quantum chip with nine qubits.

both ends - using atomic clocks, for example. Modern classical communications rely heavily on satellite technology. Transferring quantum information to the ground from a satellite in a low Earth orbit (at an altitude of about 500 kilometres) is within the reach of current technology, thanks to ground-based telescopes with metre-sized apertures that can collect most of the light from a beam that has spread out during its passage from a satellite. But transferring quantum information from the ground to a satellite, or between satellites, is more difficult because satellites cannot carry large optics.

By contrast, it is easy to measure all Bell-detection outcomes for continuousvariable systems such as electric fields, using only simple linear optics and standard photodetectors. Such systems can convey simultaneously the equivalent of many qubits, which makes them appealing for use in high-rate quantum communications ${ }^{6}$. But because the range of distances over which they can teleport is currently limited, continuous-variable systems are used less frequently than are qubits.

Approaches are needed that combine the best features of discrete variables (teleportation over long distances) with those of continuous variables (fast, deterministic teleportation). Teleportation that uses such combinations has been demonstrated over table-top distances. One experiment ${ }^{7}$ combined a discrete qubit with a continuousvariable entangled source to teleport quantum information deterministically. Further studies should help both to extend the distances covered by these experiments and to integrate qubits with other types of quantum technologies, including quantum memories for the storage of teleported information.

Studies of hybrid technologies will require greater collaboration and interaction between teams with different specializations.

\section{QUANTUM INTERNET}

One of the greatest challenges for implementing a globally distributed quantum computer or a quantum internet is entangling nodes across the network ${ }^{8}$. Qubits can then be teleported between any pair and processed by local quantum computers.

Ideally, nodes should be entangled either in pairs or by creating a large, multientangled 'cluster state' that is broadcast to all nodes. Cluster states that link thousands of nodes have already been created in the laboratory ${ }^{9}$. The challenges are to demonstrate how they might be deployed over long distances, as well as how to store quantum states at the nodes and update them constantly using quantum codes.

Quantum networks require memories to store quantum information, ideally for hours - shielding it from unwanted interactions with the environment. Such memories are 


\section{QUANTUM TELEPORTATION}

A signal, such as the polarization state of a photon, can be teleported from one place to another using entangled photons and quantum measurements.

(Physicists often call the sender Alice and the receiver Bob.)

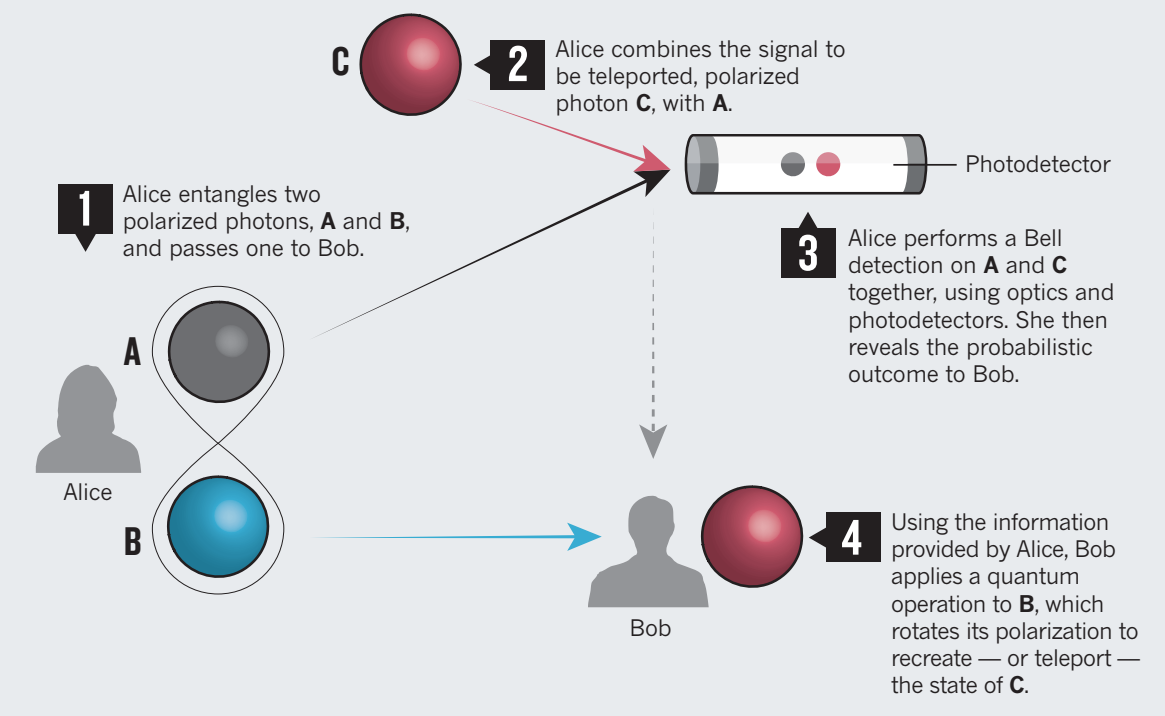

needed for quantum computing at nodes and also for the faithful, long-distance distribution of entanglement through quantum repeaters.

Quantum memories need to convert electromagnetic radiation into physical changes in matter with near-perfect read-write fidelity and at high capacity. 'Spin ensembles' represent one type of quantum memory. Ultracold atomic gases consisting of about one million atoms of rubidium can convert a single photon into a collective atomic excitation known as a spin wave. Storage times are approaching the 100 milliseconds required to transmit an optical signal across the world.

Solid-state quantum memories are even more appealing. Crystalline-solid spin ensembles - created by inserting lattice defects known as nitrogen-vacancy centres into diamonds, or by doping rare-earth crystals - can remain coherent for hours at cryogenic temperatures.

Superconducting qubits, which are defined by physical quantities such as the charge of a capacitor or the flux of an inductor, interact within a quantum processor by releasing and absorbing microwave photons. For the successful integration of solid-state quantum memory, reversible storage and retrieval of quantum information must be made possible. This will require an efficient interface between the microwave photons and the atomic spins of a solid-state quantum memory that is attached to the processor. If successful, this hybrid technology would become the most promising architecture to be scaled up into a large, distributed quantum computer.

The incorporation of superconducting processors into a quantum internet also requires that locally processed and stored microwave photons interface with optical signals (often carried in fibres), which are the most robust carriers of quantum information over long distances. A hybrid solution, known as an optomechanical quantum transducer, is emerging ${ }^{10}$. These devices exploit nanomechanical oscillators (such as
"The

development

of a quantum

internet needs

investment on

a much larger

scale."

microscopic vibrating mirrors) to transform optical photons into microwave photons, and vice versa. But their efficiency must be improved to ensure that qubits are not lost during the conversion process and that all of their quantum features are preserved. The conversion efficiency is currently about $10 \%$ (ref. 10).

The next 15 years could see the construction of a hybrid-technology quantum internet. In the vision we outline, superconducting quantum processors will be integrated with solid-state memories for local quantum storage and then augmented with microwaveoptical transducers for long-distance optical communication. After two remote nodes have been connected in this way, entanglement can be distributed between distant quantum processors to enable teleportation.

\section{NEXT STEPS}

To make this vision a reality, the following three steps should be priorities for quantum teleportation science.
First, more research - theoretical and experimental - is needed at the interface between discrete and continuous variables; dedicated conferences would help. This would enable us to blend these currently distinct approaches to exploit the best of both. Satellite experiments with polarization qubits should be pursued, and continuousvariable teleportation should be extended beyond the lab for communication within cities using free space or optical fibres.

Second, the most successful technologies will be those that integrate data communication and data storage. We need to invest in the development of a more efficient interface between superconducting quantum processors and solid-state quantum memories. This would improve the performance of the storage and retrieval of microwave photons. A tangible next step could be on-chip teleportation between a superconducting qubit and a nitrogen-vacancy centre in a local quantum memory.

Third, investment should be made in technologies that show promise of scalability. For example, microwave-optical transducers that can efficiently connect microwave photons with optical photons on a chip for longdistance quantum communication should be designed and integrated. Two remote chips could be linked by paired transducers, paving the way for long-distance quantum teleportation between superconducting qubits.

These steps will necessitate a closer interaction between researchers in superconducting quantum computing and those who are developing long-distance quantum optical communications. Industry must also be involved, especially multinational corporations that are leaders in computer hardware and telecommunications. Quantum technology is attracting private stakeholders, but the development of a quantum internet needs investment on a much larger scale.

Stefano Pirandola is reader and Samuel L. Braunstein is professor in the Department of Computer Science at the University of York, UK.

e-mails: stefano.pirandola@york.ac.uk; sam.braunstein@york.ac.uk

1. Bennett, C. H. et al. Phys. Rev. Lett. 70, 18951899 (1993).

2. Pirandola, S., Eisert, J., Weedbrook, C., Furusawa, A. \& Braunstein, S. L. Nature Photon. 9, 641-652 (2015).

3. Ma, X.-S. et al. Nature 489, 269-273 (2012).

4. Weedbrook, C. et al. Rev. Mod. Phys. 84, 621-669 (2012).

5. Knill, E., Laflamme, R. \& Milburn, G. J. Nature 409, 46-52 (2001).

6. Pirandola, S. et al. Nature Photon. 9, 397-402 (2015).

7. Takeda, S., Mizuta, T., Fuwa, M., van Loock, P. \& Furusawa, A. Nature 500, 315-318 (2013).

8. Kimble, H. J. Nature 453, 1023-1030 (2008)

9. Yokoyama, S. et al. Nature Photon. 7, 982-986 (2013).

10.Andrews, R. W. et al. Nature Phys. 10, 321-326 (2014). 